\title{
Amygdalar Models of Neurological and Neuropsychiatric Disorders
}

\author{
Trevor H. Gilbert
}

Additional information is available at the end of the chapter

http://dx.doi.org/10.5772/50244

\section{Introduction}

A basic underlying propensity of the central nervous system is the capacity to change. Neuroplasticity refers to the adaptive capacity of the brain to undergo alteration of structural organization and functioning in response to environmental change [1]. While this appears to be a very useful process, potential extremes of such plasticity can result in various forms of neurological and neuropsychiatric anomalies. Therefore, it appears that the same mechanisms that are important in adaptive activity, like learning and memory, can provide the basis for abnormal discharge. This being the case, how is it that the normal healthy brain becomes transformed into an abnormal brain? Neuroscientists use animal models to help them uncover the underlying neural mechanisms.

While animal research has revealed extensive information on the cellular, synaptic, and pathological mechanisms of neurological and neuropsychiatric processes, many unanswered questions remain. For instance, there is no neuropsychiatric disorder that is adequately understood from the level of clinical manifestations to the underlying cellular and molecular mechanisms. Because of the ethical barriers to using humans and the inherent limitations of using excised human tissue, inquiry into neurological and neuropsychiatric disorders has necessarily relied upon experimental models. For example, in vivo animal models have been indispensable for the testing of anticonvulsant drugs, for determining some of the basic mechanisms of seizure expression, and for determining changes in functional and morphological properties [2-4].

Animal models have been, and will continue to be invaluable for: 1) modeling the clinical condition, 2) determining the basic mechanisms underlying the genesis and progression of the disease, and 3) screening potential pharmacological agents. While an ideal model would possess properties identical to all aspects of the particular human condition, this is most likely unattainable. The characteristics of a valuable model should possess a substantial 
degree of similarity to, at least some aspect, of the disorder of interest [5,6]. Accordingly, a good animal model should be able to provide new understanding into the disorder it models and respond in a reproducible and predictable manner. For example, the kindling model, depending on the animal species, possesses many of these characteristics and is an excellent model for certain clinical disorders, their underlying mechanisms, and as a screening tool for newer pharmacological agents [3,4].

An understanding of the brain circuitry that underlies normal and adaptive behaviors provides a foundation for clarifying the pathology and pathophysiology of psychiatric disorders. The key structures and pathways associated with abnormal behaviors have been framed through a weighty historical record of anatomical observations. Neuroscience has been gradually unveiling the neural networks that subserve the functional domains of motivation, emotion, and cognition, central tenets to psychiatry. The convergence of findings from the multiplicity of research methods and techniques across both animal and human studies has provided the neurocircuitry framework pertinent to neurological and neuropsychiatric diseases.

A brain structure that receives substantial scientific interest today is the amygdala. The amygdalae are a group of nuclei located deep within the medial temporal lobe of the brain [7]. It has a wide range of connections with other brain regions, allowing it to participate in a wide variety of behavioral functions. Considered to be part of the limbic system, the amygdala performs an important role in the processing and memory of emotional reactions. Structural and functional changes within the amygdala are associated with a broad range of psychiatric conditions in humans, such as anxiety disorders [8], depression [9], schizophrenia [10], and autism [11].

This chapter will review the various experimental models employed to gain insight into the underlying mechanisms of key neurological and neuropsychiatric disease states, with a focus on the amygdalae. In particular, this chapter will examine models of brain plasticity, models of epilepsy, fear and anxiety, affective disorders, neurodevelopmental disorders, and neurodegenerative disorders.

\section{The amygdala}

The amygdaloid complex is a large subcortical structure located in the rostromedial part of the temporal lobe in front of the hippocampus and underneath the uncus of the parahippocampal gyrus (i.e., entorhinal cortex). Some of the most prominent pathways to the amygdaloid complex come from cortical areas in the temporal and frontal lobes. Its components are morphologically and histochemically heterogenous, as are its functions and connections with other brain regions [12]. The amygdala has close interconnections with both cortical and subcortical areas. The cortical areas include temporal (perirhinal and entorhinal), frontal, insular, and cingulate association areas and the subcortical areas include brainstem, hypothalamus, thalamus, hippocampus, and claustrum [12,13].

The many components of the amygdaloid complex can be grouped into three principal divisions: the basolateral, cortical, and centromedial nuclear groups. The largest part of the 
amygdala is the basolateral nuclear group. It is closely related to many cortical areas in the prefrontal, temporal, insular, and occipital regions and is reciprocally connected to them. The basolateral amygdala projects to the striatum and also has close relations with the thalamus. The cortical, or olfactory, amygdala receives olfactory input directly from the olfactory bulb and indirectly from the olfactory cortex. The olfactory amygdala in turn projects to the centromedial amygdala and the hypothalamus. The centromedial amygdala receives direct input from the cerebral cortex, but is largely restricted to fibers from the hippocampal formation, insula, and orbitofrontal cortex. Because the amygdala is a bidirectional pathway that can relay information between association cortices and subcortical structures it is in an optimal position to simultaneously influence excitability of numerous brain regions at any given time $[12,13]$.

\section{Kindling as a model of brain plasticity}

An excellent model for investigating brain mechanisms associated with neurological and neuropsychiatric disorders is kindling. It was Graham Goddard who first acknowledged the potential importance of repeated stimulation to the brain as a useful scientific tool. Goddard observed that some of his rats began to develop seizures, even with invariant stimulation [14]. He and his colleagues studied the phenomenon in more detail $[15,16]$ and subsequently referred to the development of stimulation-induced convulsions as "kindling," analogous to starting a fire with an initially benign stimulus [14]. The original kindling work identified the amygdala as a critical site due to its reliable and rapid kindling progression. Although subsequent research has revealed other salient sites, the amygdala is the most frequently targeted structure for kindling studies due to its proximal connections with both cortical and subcortical areas.

In its original description, kindling refers to the progressive development of epileptiform activity and associated behavioural convulsions in response to low-intensity electrical stimulation of the brain [15,16]. Kindling is generally considered to be the best available model of temporal lobe epilepsy and is particularly well suited for experimental study because precise control can be implemented over experimental conditions [17]. Progression of behavioural and electrographic activity is gradual enough to allow for particular manipulation at various phases of the kindling process $[18,19]$. There are several significant characteristics that support kindling's validity as a clinical model, especially for epilepsy. For example, the EEG patterns are similar between the experimental and human conditions, the anticonvulsant pharmacology is similar, and the cognitive and behavioural abnormalities associated with each condition are also similar $[17,20]$. These characteristics, along with kindling as a model of epilepsy, will be described in more detail below.

Although most kindling investigations have utilized rats, kindling has been described in many other animals, including amphibians, reptiles, mice, gerbils, cats, monkeys, baboons [21], and guinea-pigs $[3,4,22,23]$. It would be surprising to find a phenomenon that bridges vertebrates, including amphibians, reptiles, and mammals that would not include humans. Indeed, it has also been reported that a human has been kindled [24]. This highlights the 
applicability of the animal model for humans. Although kindling was originally defined as resulting from electrical stimulation, it now collectively refers to the eventual development of persistent brain activity following repeated exposure to a stimulus - this can be in the form of stimulus trains (electrical activity) or chemical agents. Although kindling is usually employed as an experimental model of human temporal lobe epilepsy, kindling in its simplest dimension is a model of neural plasticity [14]. In particular, the realization that persistent changes in brain function occur in response to an invariant stimulus has led many researchers to believe that such mechanisms may be similar to those underlying learning [16,25].

\section{Kindling as a model of neuropsychiatric illness}

As mentioned, kindling is commonly used as a model of epilepsy, but many researchers suggest that kindling shows a number of attributes that are similar to neuropsychiatric disorders. In particular, kindling is useful in understanding the long-term and developing patterns of non-seizure related disorders [26]. Kindling is associated with a progressive increase in behavioural and physiological responsivity and a decrease in threshold for an event (e.g., a seizure) and can eventually result in a spontaneous event (i.e., spontaneous seizure). Because the seizure is used as a convenient endpoint for behavioural and physiological responsivity, it is believed that the kindling model can be used to understand the progression of various neuropsychiatric disorders [27]. The justification is as follows. The disorders are initially expressed with minor behavioural and physiological changes, to more complete symptomatology, and then finally to the spontaneous elicitation of the disorder.

As mentioned previously, the amygdala is a bidirectional pathway that can relay information between association cortices and subcortical structures, and is therefore optimally positioned to simultaneously influence the excitability of numerous brain regions at any given time. It has become increasingly perceptible that structural and functional changes in the amygdala, and associated limbic circuitry, are linked to a variety of clinical conditions in humans. It is highly conceivable that the amygdala has been connected to various neuropsychiatric and neurodevelopmental disorders more than any other brain region.

The recurrent nature of many neuropsychiatric disorders has been documented for nearly as long as the disorders have been investigated [28]. Although it should not be surprising that there are many variations within patients, the general course of untreated illnesses tends to be predictable. There is a characterization of a diminished healthy period between episodes, and a transition from induced events (e.g., personal losses and stressors) to those periods that occur more spontaneously. Perhaps the best illustration of this is seen in bipolar disorder - minor episodes give way to major episodes. In the beginning there are isolated and intermittent events to more continuous rhythmical events, and then to the rapid cycling variety [29]. In context of the kindling model, the course of the illness is progressive in that there is threshold lowering, and a movement from triggered episodes to spontaneous episodes. Stated in a theoretical framework, kindling provides a model for conceptualizing physiological and behavioural abnormalities that progress in severity in response to the same inducing stimulus over time. 
In panic disorders, it is apparent that some patients progress from highly intermittent attacks that are precipitated by a cue, to those that begin to occur more frequently and spontaneously. In addition, some patients become increasingly more paralyzed by agoraphobia, which may increase to the point of complete incapacitation. In the case of cocaine-related panic attacks, some individuals with a history of anxiety-free episodes with repeated administration can suddenly experience a full-blown panic attack. Furthermore, it appears that some users can display panic attacks in the absence of continued drug administration [30]. Again, it is kindling's progressive feature with a threshold lowering and the move from induced to spontaneous events, that models panic disorder.

In obsessive compulsive disorder (OCD), initial obsessions and compulsions often increase in severity and lead to incapacitation. This progressive nature can be modeled by kindling. In post-traumatic stress disorder (PTSD), individuals who have experienced prior traumatic events also appear more likely to suffer from PTSD upon repeated exposure to trauma $[31,32]$. Additionally, it appears that repeated inducing events result in more severe and long-lasting consequences than isolated events. It appears that there may be a progression in the emergence of flashbacks, which are initially triggered by cues linked to the original event and occur more spontaneously. The decreases in threshold observed in kindling may model greater episode recurrence in this disorder.

Kindling has also been used to study emotionality [33]. It has been shown that the kindling in rats [34] and cats [35] can result in enhanced reactivity and aggressive behaviour. Other more enduring behavioural changes that have also been reported following limbic kindling have included diminished predatory attack behaviour in cats [36] and decreased ability to learn passive avoidance in rats [37]. More recently, it has been reported that repeatedly inducing seizures in the amygdala of rats can produce various changes in emotional responding, particularly those involving fear and anxiety [38].

It may be argued that these neuropsychiatric phenomena are perhaps only analogous, and not homologous, to kindling. However, because of many phenomenological similarities, kindling may be pertinent to the understanding of the long-term and progressive nature of such disorders.

\section{Epilepsy}

The epilepsies comprise a diverse collection of disorders that affect approximately $1 \%$ of the general population [39]. Epilepsy is a chronic brain disorder, characterized by recurrent seizures due to excessive discharge of neurons, with a spectrum of severity ranging from mild and benign to severe and intractable. Epilepsy involves a temporary electrical disturbance in the brain that can produce effects ranging from momentary loss of consciousness to full-blown generalized seizures that involve involuntary jerking of the muscles. The various epilepsies affect an estimated 40-50 million people worldwide, signifying that epilepsy is a serious neurological disorder and a significant health problem in our society. Much of our understanding about epilepsy has come from research on animals. 
Although research has revealed much information about the cellular and synaptic mechanisms of seizures and epileptogenesis, many unanswered questions remain. Animal models have been and will likely continue to be invaluable for determining the underlying mechanisms of the genesis, expression, and propagation of seizures, as well as for the screening of new anticonvulsant drugs (ACDs). The identification of new ACDs remains the most obvious and important avenue for therapeutic advancement. Because all new ACDs are tested on animal models, it seems prudent to test these new drugs on appropriate seizure models so as not to discard a potentially valuable drug because it was inadvertently tested in an unsuitable model. Accordingly, an important consideration in ACD development is the choice of an appropriate animal model for in vivo drug testing [3,4].

Experimental testing of novel ACDs requires animal models that are predictive in anticonvulsant efficacy against specific seizure types and in terms of adverse or toxic effects at anticonvulsant doses. Models that mimic the symptoms, natural history, etiology, and response to therapy of human seizures are crucial. The most commonly used animal models are the maximal electroshock (MES) test and the pentylenetetrazol (PTZ) seizure test. While both these models are thought to represent valid models for generalized tonic-clonic (grand mal) seizures and generalized absence (petit mal) seizures, respectively [40], they are not valid models for partial seizures. Unfortunately the anticonvulsant activity of common ACDs in these models are not effective against partial seizures, the most common type of all epileptic seizures. Therefore, valid models of partial seizures should be used to test the anticonvulsant efficacy against partial epileptic activity [3,4,41].

As introduced previously, kindling is generally considered to be the best available model of human partial epilepsy, and is particularly convenient for experimental study because precise control can be implemented over experimental conditions. The progression of behavioural and electrographic activity is gradual enough to allow for specific manipulations at various phases of the kindling process. There are several significant characteristics that support kindling's validity as a model for partial epilepsy: (1) the EEG patterns of hippocampal and amygdala kindled seizures are similar to human complex partial seizures; (2) the behaviour associated with initial and later seizures is similar to those of human complex partial (limbic or temporal lobe) seizures; (3) the anticonvulsant pharmacology of kindled and human partial seizures are markedly similar; (4) the occurrence of heterogenous interictal spike transients are common to the kindling model and human partial epilepsy; (5) the occurrence of spontaneous seizures in kindled animals; (6) kindling can be produced in subhuman primates and kindling-like effects have also been observed in humans; and (7) cognitive and behavioural abnormalities have been reported to occur in kindled animals and in human epileptics $[17,20]$.

Although amygdalar kindling is widely used in rat models, its application to guinea-pig models provides a unique vehicle. With its important interconnections, relative ease of anatomical location, and reliable epileptiform correlates, the amygdala remains an important target for study. With repeated stimulation, guinea-pigs show some initial growth of the electrographic indices and behavioural seizures. However, unlike rats, guinea-pigs become arrested at partial and complex partial seizures and do not progress to 
fully generalized convulsions during regular single-site kindling [22,23]. This suggests that guinea-pig kindling may be a better model for human complex partial epilepsy because the majority of humans with complex partial epilepsy also do not progress to fully generalized convulsions. Furthermore, guinea-pig seizures mimic human seizure behaviour and have a similar EEG pattern for both ictal and interictal events $[3,4]$.

Because guinea-pig kindling shares many characteristics with human partial epilepsy it will most likely continue to develop as an excellent model of the disorder. Seizures kindled in guinea-pigs echo that of human seizure expression, have a similar EEG pattern, display more severe seizures when multiple foci are present, and share a close correspondence in anticonvulsant pharmacology. Moreover, guinea-pigs slowly manifest seizures and become arrested at partial seizures, a consistent observation associated with many species of primates [42], including humans [20].

The guinea-pig with its sustained period of partial epileptic activity provides certain advantages over rats and mice as a model system for human partial epilepsy. There is a striking correlation between drugs effective against guinea-pig kindled seizures and drugs effective against partial seizures in humans. This correlation strengthens the validity of guinea-pig kindling as a model of the human condition, as it seems unlikely that a drug that is ineffective against guinea-pig kindled partial seizures will be efficacious against partial seizures in humans. Accordingly, examining the efficacy of experimental drugs in this model would seem to be a beneficial step before undertaking expensive human clinical trials. However, if examination of secondarily generalized seizures was of primary interest, then rat kindling would be the preferential model due to their speedy seizure progression and development of tonic-clonic seizures.

Future pharmacological studies should be implemented in the guinea-pig model of partial epilepsy to determine the effectiveness of other existing and newer ACDs to further elucidate the value of this model for the screening of prospective ACDs. Such studies should extend detailed acute and chronic pharmacokinetic analysis to the test drugs in an attempt to understand the drug's absorption and elimination parameters. Concurrent objective behavioural assessment should also be carried out to determine potential adverse effects of the drugs. While general observation of the animal's behaviour is important, objective quantitative tests allow for greater inter-observer reliability and are potentially more sensitive to the behavioural impairment of an ACD. Administration of an ACD requires the optimal balance between the anticonvulsant effect and the level of behavioural toxicity. Ideally, the more efficacious drugs will have powerful anticonvulsant actions in the absence of behavioural impairment. Thus, the sedation and ataxic effects of the test drug need to be assessed over multiple doses. It is only with a more in depth inquiry into the underlying mechanisms of epilepsy that will prove to be useful in human therapeutic approaches.

\section{Anxiety disorders}

Anxiety is chronic fear that persists in the absence of any direct threat, and is a common psychological correlate of stress. When anxiety becomes so disruptive that it interrupts 
normal functioning, it may manifest as an anxiety disorder. Anxiety disorders are the most prevalent of all psychiatric disorders, in that about $17 \%$ of people suffer from an anxiety disorder at some point in their lives $[43,44]$.

The amygdala is known to play an important role in normal fear conditioning and is implicated in the pathophysiology of anxiety disorders. The amygdala may also be a target for the beneficial effects of cognitive-behavioral and medication treatments for anxiety disorders [45]. Animal models have played an important role in the study of anxiety, and in the assessment of anxiolytic properties of new psychotherapeutics. Such models typically involve animal defensive behaviors, with the assumption that defensive behaviors are motivated by fear, where fear and anxiety are identical states. Common models anxiety in animals include the elevated-plus maze test, defensive burying, and risk assessment [46].

In the elevated-plus maze test, rats are placed within a four-armed maze that is raised above the floor. Two of the arms have sides, and two do not have sides. The measure of anxiety is the proportion of time spent in the enclosed arms versus the time spent in the exposed arms. In the defensive-burying test, rats are exposed to a shock apparatus housed at one end of a test chamber. Anxiety is measured by the amount of time rats spend spraying bedding material towards the source of the shock. In the risk-assessment test, rats are briefly exposed to the presence of a cat. They quickly flee to their burrows and freeze, and then engage in a variety of risk-assessment behaviors (e.g., cautiously exploring the surface) before their normal behavior returns. Anxiety is measured by the amount of time that the rat spends in both freezing and in risk assessment behaviors. Note that these anxiety models have generally been validated by observation of anxiety reduction through the use of benzodiazepines versus non-anxiolytic drugs [47].

A primary technique for investigating the mechanisms underlying emotional associative learning is Pavlovian conditioning. Studies investigating the neural mechanisms of fear conditioning across species indicate that the amygdala has a critical function in the acquisition, storage, and expression of conditioned fear [48]. Further, research suggests that interaction between the amygdala, the ventromedial prefrontal cortex and the hippocampus supports the acquisition, storage, retrieval, and contextual modulation of fear extinction.

Neuroimaging research has helped to advance neurobiological models of anxiety disorders. In individuals with PTSD, research suggests altered emotion neurocircuitry. Emotion activation studies in these individuals have shown hyperactivation in emotion-related regions, including the amygdala and insula, and hypoactivation in emotion-regulation regions, including the medial prefrontal cortex and anterior cingulate cortex. Increased connectivity between the amygdala and insula may suggest enhanced anticipation of negative events [49].

\section{Neurodevelopmental disorders}

The amygdala has been implicated in a range of neurodevelopmental disorders. Like our entire nervous system, it seems that the amygdala is sensitive to threshold variances. Amygdalar dysregulation can result in perceptual changes related to environmental danger 
and sociality, and thereby result in dysfunctional anxiety. Across a range of studies investigating neurodevelopmental disorders, there is evidence of amygdala dysregulation from postmortem studies, structural MRI analyses, and functional MRI [50].

Schizophrenia is a disorder characterized by deteriorating ability to function in everyday life, and includes some combination of hallucinations, delusions, thought disorder, movement disorder, and inappropriate emotional expression. It attacks about $1 \%$ of individuals of all races and cultural groups, typically beginning in adolescence or early adult [43,51].

Given the substantial contribution of genetic factors to many disease conditions, an obvious way of developing animal models would be insertion of human disease-associated alleles into mice. Indeed, genetic animal models developed from highly penetrant human mutations seem like suitable candidates [52]. However, caution is required as work is ongoing to identify which of the genes, or deleted genes, are responsible for the relevant behavioral abnormalities in mouse models. While such genetic animal models made with variants of small effect may demonstrate interesting neurobiological properties, perhaps it is still too early to accept these models as mirroring the etiology of the disease [46].

Animal models of maternal infection, or environmental models, have been widely employed to look at immune involvement in schizophrenia. For example, prenatal viral infection has been used to induce behavioral and neural abnormalities [53], such as the expression of immune-related molecules (cytokines) in the brain and cerebral spinal fluid. Perhaps this represents a permanent state of brain immune dysregulation which begins during early development. Although such results related to immune involvement encourage novel therapeutic immune interventions, the role of viral infection in schizophrenia remains unclear.

Other experimental attempts have used pharmacological manipulations or lesions to produce symptoms of schizophrenia. The efficacy of dopamine receptor antagonist drugs in treating positive symptoms of schizophrenia gave rise to the deep-rooted dopamine hypothesis. Subsequently, the observation that NMDA (n-methyl-d-aspartate) glutamate receptor antagonists (e.g., phencyclidine, ketamine) produce psychotic symptoms and cognitive disturbances redolent of schizophrenia gave rise to the glutamate hypothesis [54]. Consequently, diverse animal models have been based on manipulations of these principal transmitter systems, although the putative dopaminergic or glutamatergic abnormalities in schizophrenia have not been indubitably established [55].

On the basis of evidence obtained from in vivo brain imaging, postmortem, and rodent studies, it seems likely that amygdalocortical circuitry has an important function in the pathophysiology of schizophrenia. Projections of the basolateral amygdala may be of particular importance to the induction of abnormal circuitry in schizophrenia, as their ingrowth during late adolescence and early adulthood may help to trigger the onset of illness in susceptible individuals [56]. Ultimately then, it seems that abnormalities in limbic lobe components may contribute to a dysfunctional regulation of affective responses in schizophrenics.

Autism is a complex debilitating neurodevelopmental disorder, which involves a wide range of problematic behaviours including deficits in language, deficits in perceptual and motor development, defective reality testing, and an inability to function in social situations [43]. 
The amygdala has long been a site of intense interest in the search for neuropathology in the brains of people with autism, given its role in modulating anxiety levels and social behavior. Schumann et al. [50] conducted a study where autistic adults showed a decrease in neurons in the amygdala compared to control groups. It appears that the amygdala undergoes an abnormal pattern of postnatal development that includes unusual enlargement, followed by a reduction of amygdala size, or decreased neurons, into adulthood.

Howard et al. [57] found that individuals with high-functioning autism demonstrated neuropsychological profiles characteristic of the effects of amygdala damage such as, impairment in the recognition of facial expressions of fear, perception of eye-gaze direction, and recognition memory for faces. They used MRI analysis techniques to show that the same individuals also demonstrated abnormalities of medial temporal lobe brain structure, notably bilaterally enlarged amygdala volumes, perhaps reflecting incomplete neuronal pruning in early development. These results suggest that developmental malformation of the amygdala may underlie the social-cognitive impairments characteristic of autism.

Other studies have reported that amygdala lesions in isolation are not sufficient for producing autistic symptoms. Instead, it may be abnormal connectivity between the amygdala and other structures that contributes to autistic symptoms at a network level [58]. Dziobek et al. [59] used imaging techniques to measure fusiform gyrus cortical thickness and to measure amygdala volumes, and suggest that dysfunction of the amygdalafusiform system might represent a crucial pathophysiological mechanism of autism.

With recent findings linking multiple genes to autism spectrum disorders, novel rodent mutants with deletions, truncations, and/or overexpression of these candidate genes have been developed and studied both behaviorally and biologically. As the diagnostic criteria for autism are behavioural, phenotyping these mouse models requires behavioural assays with high relevance to each category of the diagnostic symptoms. These include tests for social interaction, communication and repetitive behaviours to test hypotheses about the causes of autism. Such mouse models hold great promise as tools for discovering effective treatments for components of autism spectrum disorders [60].

\section{Mood disorders}

Although there a continuum of mood disorders has been identified, the best documented ones are depression and mania. The primary symptoms of major depression are prolonged feelings of worthlessness and guilt, disruption of normal eating habits, sleep disturbances, a general slowing of behavior, and frequent thoughts of suicide. Mania is the polar opposite from depression, and is characterized by excessive euphoria. The affected person is typically hyperactive and often formulates grandiose plans. The periods of mania often fluctuate into states of depression and back again into mania. This condition is referred to as bipolar disorder (BD). There is a high incidence of affective disorders in Western societies, with about $5 \%$ of the population suffering from major depression, and $1-2 \%$ suffering from bipolar disorder [51,61]. 
Several chronic stress procedures have been employed in various animal models [46]. Typically, chronic mild or chronic unpredictable stress involves subjecting normal rodents to a series of repeated physical stresses (e.g., restraint, foot shock, cold temperature) over a period of weeks. The animals show signs of anhedonia (e.g., reduced sucrose preference), which can be reversed by chronic administration of antidepressant medications. Further, repeated stress results in hyperexcitability in the basolateral amygdala in rodents [62], suggesting a mechanism that might produce pathological amygdala activity in depression.

Chronic social defeat stress involves subjecting rodents to repeated cycles of social subordination, after which the rodents show a range of depression symptom (e.g., anhedonia, social withdrawal) which can be reversed by chronic antidepressants [63]. It appears that chronic social defeat also induces a metabolic syndrome in mice characterized by weight gain, and insulin and leptin resistance, a finding that is consistent with homeostatic abnormalities observed in human depression [64].

Another model of depression is based on early life stress. Maternal separation in rat pups induces life-long behavioral and neuroendocrine abnormalities in the pups, some of which can be reversed by antidepressant medications [65]. In addition, it was recently found that prolonged exposure (weeks to months) of adult rodents to social isolation induces anhedonia, which can also be treated effectively with chronic antidepressant medication [66].

Patients with mood disorders manifest abnormalities of morphology in several medial prefrontal network and limbic structures. A large body of human data from functional and structural imaging, as well as analysis of lesions and histological material indicates that this system is centrally involved in mood disorders. Neural models of depression suggest that dysfunction within the medial network and related limbic structures underlie the disturbances in emotional behavior and other cognitive aspects of the major depressive syndrome [62].

The most often used model of mania involves treating normal rodents with psychostimulants (e.g., cocaine or amphetamine). The repeated administration of the drugs causes sensitization of the acute locomotor-activating effects of the drugs, which in turn can be reduced by lithium or valproate, two chief treatments for human bipolar disorder [67]. Although these are intriguing findings, caution with this model is warranted. It remains unclear that the molecular and cellular changes associated with psychostimulant-induced sensitization have direct equivalence with the pathophysiology of bipolar disorder.

As mentioned previously, chronic stress is known to induce hyperactivation of amygdala, enhancing amygdala-dependent unlearned fear, fear conditioning, and aggression. Similarly, many of the symptoms experienced by patients with bipolar disorder appear to be associated with abnormalities in emotional processing, and an enlargement of the amygdala has been described as a prominent abnormality in BD. Abnormal age related increases in the amygdala volume have been found in bipolar adolescents. In addition to structural changes in this circuitry, functional neuroimaging studies indicate increased activity in the amygdala during acute mood episodes [68].

Other imaging studies have looked at changes in amygdalar regulation in BD. Bechdolf et al. [69] investigated adolescents and young adults with BD prior to the onset of first episode 
mania. They found that amygdala and insula volume reductions are present prior to the onset of first episode mania, a reduction consistent with the amygdala's central role in emotion processing, such as associating stimuli with emotional responses, enhancing memory consolidation and influencing perception of stimuli. Another study found increased amygdalar activation in BD patients, suggesting an exaggerated response to negative emotional stimuli [70].

\section{Neurodegenerative diseases}

Alzheimer's disease (AD) is a devastating degenerative brain disorder related to aging that first appears as progressive memory loss and later develops into generalized dementia. About $10 \%$ of the general population over the age of 65 suffers from the disease, and the proportion is about $35 \%$ in those over 85 [43].

$\mathrm{AD}$ brains are characterized by the presence of amyloid plaques and neurofibrillary tangles. Plaques are clumps of scar tissue composed of degenerating neurons and amyloid protein, and the tangles are knots of protein in the cytoplasm. Although plaques and tangles are observed throughout the cerebral cortex, they are most prevalent in medial temporal lobe structures such as the amygdala, entorhinal cortex, and hippocampus [71]. The amygdala of $\mathrm{AD}$ patients shows significant shrinkage, distortion and loss of neurons as well as extensive gliosis [72,73]. Additionally, the extent of the amygdalar atrophy correlates positively with the degree of emotional memory impairment [71].

Knafo et al. [74] describes the neuropathological changes in the amygdala and alterations in emotional memory of transgenic AD mouse models. They suggest that the morphological changes found in the neurons of the lateral nucleus of the amygdala may contribute to the impaired auditory fear conditioning observed in this model. Further, they note that AD patients also show marked impaired fear conditioning, indicating that deficits in nondeclarative memory is common to both $\mathrm{AD}$ patients and the transgenic mice. It seems then that the behavioral features detected in this AD model are similar to those found in AD.

Parkinson's disease (PD) is a disorder of the motor system correlated with the loss of dopamine in the brain and characterized by tremors, muscular rigidity, and reduction in voluntary movement. Parkinson's disease is a movement disorder of middle and old age that affects about .5\% of the population [61]. PD is associated with widespread neuronal degeneration and the presence of cortical and brainstem Lewy bodies (clumps of proteins).

Bouchard et al. [75] bolstered evidence for a linkage between PD and volumetric decreases in limbic system structures. Through MR imaging, they found specific and predictable correlations between the age and sex of a PD patient and the degree of volumetric loss in the hippocampus and amygdala.

Freichel et al. [76] used a transgenic mouse model to assess the behavioral and structural implications of mutant $\alpha$-synuclein ( $\alpha \mathrm{SYN})$ expression, a neuronal protein. $\alpha \mathrm{SYN}$ inclusions constitute the hallmark lesions of a number of neurodegenerative diseases, including PD and dementia with Lewy bodies. Using fear conditioning, active avoidance, and water-maze 
tests, they assessed transgenic mice across a 12 month span for cognitive impairment and possible dementia. The mice showed $\alpha$-synucleinopathy in several brain regions, including the central nucleus of the amygdala, which is involved in cognitive behavior of mice, and is susceptible to $\alpha S Y N$ pathology in human patients.

In a clinico-pathological study looking at postmortem PD brains, Kalaitzakis et al. [77] assessed the correlation between various presentations of PD and specific anatomical and pathological correlates, including $\alpha \mathrm{SYN}$, tau, and amyloid deposits in regions associated with cognition. They found that the amygdala is specifically burdened with $\alpha S Y N$ in cases of PD with dementia and also was strongly associated with visual hallucinations. These findings support the literature on the phenomenology of hallucinations in neurodegenerative diseases and the link between such hallucinations and irregularities in the limbic system.

Huntington's disease (HD) is a rare degenerative disease of the central nervous system that affects about $.01 \%$ of the population. Huntington's disease is a progressive motor disorder of middle to old age, has a strong genetic basis, and is associated with severe dementia [61].

The last 10 years or so have seen a significant generation of various transgenic models of HD. In particular, a rat model exhibits prominent cellular accumulation of huntingtin, a pathological hallmark of $\mathrm{HD}$, in addition to exhibiting ongoing emotional changes [78]. It appears that this model will be valuable in interpreting various psychiatric aspects of HD.

Further, Faure et al. [79] describe emotional blunting and hypersensitivity to negative emotional situations in symptomatic transgenic HD, and suggest that some of these symptoms may be related to amygdala dysfunction. In particular, they propose that functional alteration in brain circuits, with a possible deficit in frontal control, such as shrinkage of the central nucleus of the amygdala, may be responsible for these emotional disorders.

\section{Discussion}

Animal research has uncovered considerable information on the cellular and synaptic mechanisms of neurological and neuropsychiatric processes, however, many questions remain. Unfortunately there is no neuropsychiatric disorder that is effectively understood from the level of clinical manifestations to the underlying cellular and molecular mechanisms. Given the ethical and practical limitations to human experimental biology and neuropsychiatry, animal models will almost certainly be a necessary aspect of progress.

The development of valid and effective animal models for neurological and neuropsychiatric disorders represents a significant challenge. However, despite these challenges, model systems are necessary for understanding disease pathophysiology in order to allow for the development of appropriate treatments. A good animal model should be able to provide new understanding into the disorder it models and respond in a reproducible and predictable manner. Other practical characteristics of the model that must be taken into account include cost, ease of maintenance, genetic uniformity, and amenability to behavioural testing. 
Neuroscience has focused significant attention to the amygdala, unmasking its structure, function, and physiological mechanisms, across both animals and humans. It has become more and more evident that structural and functional changes in the amygdala are associated with a wide variety of clinical conditions in humans. Perhaps more than any other brain region, the amygdala has been linked to numerous neuropsychiatric, neurodevelopmental, and neurological disorders, and therefore a potential target for therapeutics to alleviate associated symptoms. The amygdala is an integral part of a neural system that evolved early in order to detect environment dangers and modulate subsequent responses, a role which can profoundly influence human behavior. Notwithstanding this systematic progress, much remains unknown, but the foundational knowledge obtained has provided a solid grounding in which to build upon in future work.

\section{Author details}

Trevor H. Gilbert

Centre for Psychology, Faculty of Humanities \& Social Sciences, Athabasca University, Canada

\section{Acknowledgement}

The author wishes to acknowledge the excellent research contributions of Jenna Hildebrandt, Johanna MacDonell, and Rory St. Clair.

\section{References}

[1] Shaw C, McEachern J (2001) Toward a theory of neuroplasticity. London, England: Psychology Press.

[2] Fisher RS (1989) Animal models of the epilepsies. Brain Research Reviews. 14: 245-278.

[3] Gilbert TH, Bharadia V, Teskey GC (2001) Conventional anticonvulsant drugs in the guinea-pig kindling model of partial seizures: effects of acute phenytoin. Exp. Brain Res. 140: 469-478.

[4] Gilbert TH, Teskey GC (2007) Effects of conventional anticonvulsant drugs during repeated administration in amygdala-kindled guinea-pigs. Exp. Brain Res. 178: 115-125.

[5] Nestler EJ, Hyman SE (2010) Animal models of neuropsychiatric disorders. Nature Neuroscience. 13: 1161-1169.

[6] Fernando AB, Robbins TW (2011) Animal models of neuropsychiatric disorders. Annu. Rev. Clin. Psychol. 7: 39-61.

[7] LeDoux JE (2000) Emotion circuits in the brain. Annu Rev Neurosci. 23: 155-184.

[8] Rauch SL, Shin LM, Wright CI (2003) Neuroimaging studies of amygdala function in anxiety disorders. Ann NY Acad Sci. 985: 389-410.

[9] Price, JL, Drevets, WC (2010) Neurocircuitry of mood disorders. Neuropsychopharmacology Reviews. 35: 192-216.

[10] Benes F (2010) Amygdalocortical circuitry in schizophrenia: From circuits to molecules. Neuropsychopharmacology Reviews. 35: 239-257. 
[11] Dziobek I, Bahnemann M, Convit A, Heekeren HR (2010) The role of the fusiformamygdala system in the pathophysiology of autism. Arch Gen Psychiatry. 67(4): 397-405.

[12] Amaral DG, Price JL, Pitkanen A, Carmichael ST (1992) Anatomical organization of the primate amygdaloid complex. In: Aggleton JP (ed) The Amygdala: Neurobiological Aspects of Emotion, Memory, and Mental Dysfunction. Wiley-Liss Inc., New York, pp1-66.

[13] Kandel ER, Schwartz JH, Jessell TM (2000) Principles of neural science (4 $4^{\text {th }}$ ed). Appleton \& Lange, New Jersey.

[14] Corcoran ME (1988) Characteristics and mechanisms of kindling. In: Kalivas P, Barnes C (eds) Sensitization of the Nervous System. The Telford Press, Caldwell, NJ, pp 81-116.

[15] Goddard GV (1967) Development of epileptic seizures through brain stimulation at low intensity. Nature. 214: 1020-1021.

[16] Goddard GV, McIntyre DC, Leetch CK (1969) A permanent change in brain function resulting from daily electrical stimulation. Experimental Neurology. 25: 295-330.

[17] Sato M, Racine RJ, McIntyre DC (1990) Kindling: basic mechanisms and clinical validity. Electroencephalography and Clinical Neurophysiology. 76: 459-472.

[18] Racine RJ (1972a) Modification of seizure activity by electrical stimulation: I. Afterdischarge threshold. Electroencephalography and Clinical Neurophysiology. 32: 269-279.

[19] Racine RJ (1972b) Modification of seizure activity by electrical stimulation: II. Motor seizure. Electroencephalography and Clinical Neurophysiology. 32: 281-294.

[20] Engel J (1998) The syndrome of mesial temporal lobe epilepsy: a role for kindling. In: Corcoran ME, Moshe SL (eds) Kindling 5. Plenum Press, New York, pp 469-484.

[21] McNamara JO (1984) Kindling: an animal model of complex partial epilepsy. Ann Neurol. 16 (suppl): S72-S76.

[22] Teskey GC, Thiessen EJ, Gilbert TH (1999) Alternate-site kindling in the guinea-pig results in accelerated seizure progression and generalization. Epilepsy Research. 34: 151-159.

[23] Teskey GC, Valentine PA, Sainsbury RS, Trepel C (1995) Evolution of afterdischarge and seizure characteristics during electrical kindling of the guinea pig. Brain Research. 672: 137-147.

[24] Sramka M, Sedlak P, Nadvornik P (1977) Observation of kindling phenomenon in treatment of pain by stimulation in thalamus. In: Sweet WH, Obdor S, MartinRodriguez JG (eds) Neurological Treatment in Psychiatry, Pain and Epilepsy, University Park Press, Baltimore, MD, pp 651-654.

[25] Cain DP (1989) Long-term potentiation and kindling: how similar are the mechanisms? Trends in Neuroscience. 12: 6-10.

[26] Bolwig TG (1989) New possibilities in neuropsychiatry. Ugeskr Laeger. 151(21):1339-1343.

[27] Post RM, Weiss SR (1998) Sensitization and kindling phenomena in mood, anxiety, and obsessive-compulsive disorders: the role of serotonergic mechanisms in illness progression. Biol Psychiatry. 44(3):193-206.

[28] Shepherd M (1998) Emil Kraepelin and modern psychiatry. Kaohsiung J Med Sci. 14(7): 395-404.

[29] Julien RM (2001) A Primer of Drug Action (9th ed). Worth Publishers. 
[30] Post RM, Weiss SR, Pert A (1987) The role of context and conditioning in behavioral sensitization to cocaine. Psychopharmacol Bull. 23(3): 425-9.

[31] Post RM, Weiss SR, Smith M, Rosen J, Frye M (1995) Stress, conditioning, and the temporal aspects of affective disorders. Ann N Y Acad Sci. 771: 677-96.

[32] Post RM, Weiss SR, Smith M, Li H, McCann U (1997) Kindling versus quenching. Implications for the evolution and treatment of posttraumatic stress disorder. Ann $\mathrm{N} Y$ Acad Sci. 821: 285-95.

[33] Adamec R (1990) Kindling, anxiety and limbic epilepsy: Human and animal perspectives. In: Wada JA (ed) Kindling 4. Raven Press, New York, pp 329-341.

[34] Mucha RF, Pinel JPJ (1977) Postseizure inhibition of kindled seizures. Experimental Neurology. 54: 266-282.

[35] Wada JA, Sato, M, Wake A, Green JR, Troupin AS (1976) Prophylactic effects of phenytoin, phenobarbital, and carbamazepine examined in kindling cat preparations. Archives of Neurology. 33(6): 426434.

[36] Adamec R (1975) Behavioral and epileptic determinants of predatory attack behavior in cat. Canadian Journal of Neurological Science. 2: 457-467.

[37] Boast CA, McIntyre D (1977) Bilateral kindled amygdala foci and inhibitory avoidance behavior in rats: a functional lesion effect. Physiology and Behavior. 18: 25-28.

[38] Kalynchuk LE, Pinel JP, Treit D, Kippin TE (1997) Changes in emotional behavior produced by long term amygdala kindling in rats. Biological Psychiatry. 1(4): 438451.

[39] Wiebe S (2000) Seizure control as an indicator of therapeutic usefulness. Canadian Journal of Neurological Sciences. 27(1): S97-105.

[40] Löscher W (1998) New visions in the pharmacology of anticonvulsion. European Journal of Pharmacology. 342: 1-13.

[41] Löscher W, Schmidt D (1994) Strategies in antiepileptic drug development: is rational drug design superior to random drug screening and structural variation? Epilepsy Research. 17: 95-134.

[42] Wada JA (1998) Genetic predisposition and kindling susceptibility in primates. In: Corcoran ME, Moshe SL (eds) Kindling 5. Plenum Press, New York, pp 1-14.

[43] Kolb B, Whishaw IQ (2009) Fundamentals of human neuropsychology (6 $6^{\text {th }}$ ed). Worth Publishers, New York.

[44] Shin LM, Liberzon I (2010) The neurocircuitry of fear, stress, and anxiety disorders. Neuropsychopharmacology Reviews. 35:169-191.

[45] Rauch SL, Shin LM, Wright CI (2003) Neuroimaging studies of amygdala function in anxiety disorders. Ann NY Acad Sci. 985: 389-410.

[46] Nestler EJ, Hyman SE (2010) Animal models of neuropsychiatric disorders. Nature Neuroscience. 13: 1161-1169.

[47] Davis M, Walker DL, Miles L, Grillon C (2010) Phasic vs sustained fear in rats and humans: role of the extended amygdala in fear vs anxiety. Neuropsychopharmacology Reviews. 35: 105-135.

[48] Hartley CA, Phelps EA (2010) Changing fear: the neurocircuitry of emotion regulation. Neuropsychopharmacology Reviews 35: 136-146. 
[49] Sripada RK, King AP, Garfinkel SN, Wang X, Sripada CS, Welsh RC, Liberzon I (2012) Altered resting-state amygdala functional connectivity in men with posttraumatic stress disorder. J Psychiatry Neurosci. 37(2): 1-9.

[50] Schumann CM, Bauman MD, Amaral DG (2011) Abnormal structure or function of the amygdala is a common component of neurodevelopmental disorders. Neuropsychologia. 49(4): 745-759.

[51] Kandel ER, Schwartz JH, Jessell TM (2000) Principles of neural science (4 $4^{\text {th }}$ ed). Appleton \& Lange, New Jersey.

[52] Karayiorgou M, Simon TJ, Gogos JA (2010) 22q11.2 microdeletions: linking DNA structural variation to brain dysfunction and schizophrenia. Nat. Rev. Neurosci. 11: 402-416.

[53] Patterson PH (2009) Immune involvement in schizophrenia and autism: etiology, pathology and animal models. Behav. Brain Res. 204: 313-321.

[54] Bickel S, Javitt D (2009) Neurophysiological and neurochemical animal models of schizophrenia:Focus on glutamate. Behav. Brain Res. 204: 352-362.

[55] Seillier A, Giuffrida A (2009) Evaluation of NMDA receptor models of schizophrenia: Divergences in the behavioral effects of sub-chronic PCP and MK-801. Behav. Brain Res. 204: 410-415.

[56] Benes F (2010) Amygdalocortical circuitry in schizophrenia: From circuits to molecules. Neuropsychopharmacology Reviews. 35: 239-257.

[57] Howard MA, Cowell PE, Boucher J, Broks P, Mayes A, Farrant A, Roberts N (2000) Convergent neuroanatomical and behavioural evidence of an amygdala hypothesis of autism. NeuroReport. 11(13): 2931-2935.

[58] Paul LK, Corsello C, Tranel D, Adolphs R (2010) Does bilateral damage to the human amygdala produce autistic symptoms? J Neurodevelop Disord. 2: 165-173.

[59] Dziobek I, Bahnemann M, Convit A, Heekeren HR (2010) The role of the fusiformamygdala system in the pathophysiology of autism. Arch Gen Psychiatry. 67(4): 397-405.

[60] Silverman JL, Yang M, Lord C, Crawley JN (2010) Behavioural phenotyping assays for mouse models of autism. Nature Reviews Neuroscience. 11: 490-502.

[61] Butcher JN, Mineka S, Hooley JM (2007) Abnormal psychology (13th ed). Boston: Pearson Education, Inc.

[62] Price, JL, Drevets, WC (2010) Neurocircuitry of mood disorders. Neuropsychopharmacology Reviews. 35: 192-216.

[63] Krishnan V et al (2007) Molecular adaptations underlying susceptibility and resistance to social defeat in brain reward regions. Cell. 131: 391-404.

[64] Chuang JC et al (2010) A b3-adrenergic-leptin-melanocortin circuit regulates behavioral and metabolic changes induced by chronic stress. Biol. Psychiatry. 67: 1075-1082.

[65] Meaney MJ (2001) Maternal care, gene expression and the transmission of individual differences in stress reactivity across generations. Annu. Rev. Neurosci. 24: 1161-1192.

[66] Wallace DL, Han MH, Graham DL, Green TA, Vialou V, Iñiguez SD, Cao JL, Kirk A, Chakravarty S, Kumar A, Krishnan V, Neve RL, Cooper DC, Bolaños CA, Barrot M, McClung CA, Nestler EJ (2009) CREB regulation of nucleus accumbens excitability mediates social isolation-induced behavioral deficits. Nat. Neurosci. 12: 200-209. 
[67] Einat H, Manji HK (2006) Cellular plasticity cascades: genes-to-behavior pathways in animal models of bipolar disorder. Biol. Psychiatry. 59: 1160-1171.

[68] Kapczinskia F, Vietab E, Andreazzaf AC, Freye BN, Gomesa FA, Tramontinaa J, Annaf MKS, Grassi-Oliveirag R, Post RM (2008) Allostatic load in bipolar disorder: implications for pathophysiology and treatment. Neuroscience and Biobehavioral Reviews. 32: 675-692.

[69] Bechdolf A, Wood SJ, Nelson B, Velakoulis D, Yücel M, Takahashi T, Yung AR, Berk M, Wong MT, Pantelis C, McGorry PD (2012) Amygdala and insula volumes prior to illness onset in bipolar disorder: a magnetic resonance imaging study. Psychiatry Research: Neuroimaging. 201(1): 34-39.

[70] Hulvershorn LA, Karne H, Gunn AD, Hartwick SL, Wang Y, Hummer TA, Anad A (2011) Neural activation during facial emotion processing in unmedicated bipolar depression, euthymia, and mania. Biological Psychiatry. 71(7): 603-610.

[71] Mori E, Ikeda M, Hirono N, Kitagaki H, Imamura T, Shimomura T (1999) Amygdalar volume and emotional memory in Alzheimer's disease. Am J Psychiatry. 156: 216-222.

[72] Herzog AG, Kemper TL (1980) Amygdaloid changes in aging and dementia. Arch Neurol. 37: 625-629.

[73] Vereecken THLG, Vogels OJM, Nieuwenhuys R (1994) Neuron loss and shrinkage in the amygdala in Alzheimer's disease. Neurobiology of Aging. 15: 45-54.

[74] Knafo S, Venero C, Merino-Serrais P, Fernaud-Espinosa I, Gonzalez-Soriano J, Ferrer I, Santpere G, DeFelipe J (2009) Morphological alterations to neurons of the amygdala and impaired fear conditioning in a transgenic mouse model of Alzheimer's disease. Journal of pathology. 219: 41-51.

[75] Bouchard TP, Malykhin N, Martin WRW, Hanstock CC, Emery DJ, Fisher NJ, Camicioli RM (2008) Age and dementia-associated atrophy predominates in the hippocampal head and amygdala in parkinson's disease. Neurobiology of Aging. 29(7): 1027-1039.

[76] Freichel C, Neumann M, Ballard T, Müller V, Woolley M, Ozmen L, Kahle PJ (2007) Age-dependent cognitive decline and amygdala pathology in alph $\alpha$-synuclein transgenic mice. Neurobiology of Aging. 28(9): 1421.

[77] Kalaitzakis ME, Christian LM, Moran LB, Graeber MB, Pearce RKB, Gentleman SM (2009) Dementia and visual hallucinations associated with limbic pathology in Parkinson's disease. Parkinsonism and Related Disorders. 15(3): 196-204.

[78] Petrasch-Parwez E, Nguyen HP, Löbbecke-Schumacher M, Habbes HW, Wieczorek S, Riess O, Andres KH, Dermietzel R, Von Hörsten S (2007) Cellular and subcellular localization of Huntingtin aggregates in the brain of a rat transgenic for Huntington disease. J Comp Neurol. 501: 716-30.

[79] Faure A, Höhn S, Von Hörsten S, Delatour B, Raber K, Le Blanc P, Desvignes N, Doyère V, El Massioui N (2011) Altered emotional and motivational processing in the transgenic rat model for Huntington's disease. Neurobiol Learn Mem. 95: 92-101. 